\title{
Leveraging Femtocells for Dissemination of Early Warning Messages
}

\author{
Edward Mutafungwa ${ }^{1}$, Jyrı HämäLÄIneN ${ }^{1}$ \\ 1 Department of Communications and Networks, Otakaari 5, FIN-02015, P. O. Box 3000, Teknillinen korkeakoulu \\ (Helsinki University of Technology), Espoo, Finland, edward.mutafungwa@tkk.fi
}

The main objective of any early warning system is to rapidly disseminate warning messages to specific individuals, communities or organizations in response to an imminent or oncoming hazard event (e.g. hurricane, coastal flooding, forest fire, nuclear fallout, etc.). The warning dissemination has to be executed in a manner that ensures that the message delivery is timely; the message content is accurate; understandable, and usable [GLANTZ (2003), WMO (2007)]. Therefore, all available broadcast (television, radio) and telecommunication infrastructure must be used to increase the likelihood of the warning message reaching all persons under risk and organizations expected to respond to a hazard scenario.

In recent times privatization and deregulation measures widely adopted within the telecommunications sector, resulting in, increased competition; higher efficiency; cutting-edge innovations; affordable services for users (thus increasing service penetration to hitherto unconnected citizens), and significant revenues for telecommunication service providers. As a result privately-owned telecommunication service providers are being relied upon to provide services, not just for the general public and the private sector customers, but also for government agencies and authorities. These services include both traditional (commercial) services and emergency services, such as, the early warning services addressed in this paper.

Public Land Mobile Networks (PLMN) are now considered one of the most important telecommunications infrastructures for delivery of messages originating from early warning systems. The high level of penetration of mobile handsets, subscriber mobility and the widespread adoption of mobile messaging services (in particular, the Short Message Service or SMS) make the PLMN attractive for mass dissemination of messages to targeted recipients.

However, conventional (macrocellular) PLMN are engineered to support busy hour traffic loads, rather than simultaneous messaging to a large user base, as required for early warning. As a result, warning messages delivered using mobile messaging services, such as SMS, may be excessively delayed or dropped altogether due to inadvertent network congestion or deliberate denial-of-service attacks [MENG (2007)]. Moreover, cost-constraints applied to network planning may lead to imperfect coverage, particularly in indoor environments. The methods employed to improve indoor coverage include the use of repeaters, distributed antenna systems, microcellular base stations (BSs), and the more recent femtocellular network concept [CHANDRASEKHAR (2008)].

Femtocells are characterized by simple, low cost, low transmission power and plug-and-play femto or home BSs, which are deployed indoors similar to WiFi access points. Another attractive feature of femtocells is the utilization of IP backhaul through a local broadband connection (e.g., digital subscriber lines, passive optical networks, cable modems etc.). This reduces the likelihood of congestion by avoiding possible traffic bottlenecks in macrocellular networks. Moreover, the offloading traffic from macrocellular networks to local broadband connections allows redirection of macrocell BS resources towards delivery of early warning or emergency call services to users in the area under risk but without femtocellular network coverage.

This paper presents the most significant and positive aspects of using femtocells as platform for dissemination of early warning messages. These benefits are emphasized further by pointing out the limitations in existing approaches. The possible implementation challenges for warning message delivery via femtocells are also highlighted.

\section{Literature}

Glantz, M. H. (2003): A Usable Science report on the workshop on "Early Warning Systems: Do's and Don'ts," 20-23 October 2003, Shanghai, China, report prepared February 2004

WMO (2007): WMO Multi-Hazard Early Warning Demonstration Projects, Outline for Documentation of Good Practices in Early Warning Systems, developed by the Expert Meeting on National Meteorological and Hydrological Services' Participation in Disaster Risk Reduction Coordination Mechanisms and Early Warning Systems, WMO HQ (Geneva, Switzerland), 26-28/11/2007.

Meng, X. Zefros, P., Samanta, V., Wong, S. H., \& Lu, S. (2007): Analysis of the reliability of a nationwide short message service: in Proceedings of 26th Annual IEEE Conference on Computer Communications (INFOCOM), Anchorage, Alaska, 2007, pp. 1811-1819.

Chandrasekhar, V., \& Andrews, J. G. (2008): Femtocell Networks: A Survery - IEEE Communications Magazine, Vol. 46 : p 59-67; New York 